\title{
ON THE ELASTIC DESCRIPTION OF A SPHERICAL JANUS PARTICLE
}

\author{
A.L. Kolesnikova ${ }^{1-3}$, M.Yu. Gutkin ${ }^{1-4}$, S.A. Krasnitckii ${ }^{1}$, A.M. Smirnov', \\ M.V. Dorogov' ${ }^{3}$, V.S. Serebryakova ${ }^{1}$, A.E. Romanov ${ }^{1}$ and E.C. Aifantis ${ }^{3,5}$ \\ ${ }^{1}$ ITMO University, Kronverksky pr. 49, St. Petersburg, 197101, Russia \\ ${ }^{2}$ Institute of Problems of Mechanical Engineering, Russian Academy of Sciences, 61 Bolshoj pr., V.O., \\ St. Petersburg, 199178, Russia \\ ${ }^{3}$ Togliatti State University, Belorusskaya 14, Togliatti, 445020, Russia \\ ${ }^{4}$ Peter the Great St. Petersburg Polytechnic University, Polytechnicheskaya 29, St. Petersburg, 195251, Russia \\ ${ }^{5}$ Aristotle University of Thessaloniki, University Campus, 54124, Thessaloniki, Greece
}

Received: May 07, 2018

\begin{abstract}
The elastic problem of a spherical core-shell particle with a truncated core is solved within the isotropic classical theory of elasticity. A transition to a Janus particle consisting of two unequal spherical segments with lattice mismatch is demonstrated. Elastic fields in the Janus particle are found and described in detail. The strain energy of the Janus particle in dependence on the interface coordinate is discussed.
\end{abstract}

\section{INTRODUCTION}

The Janus particles (JPs) are named after the Roman god Janus, the most ancient of Roman gods, which is the god of doors and is imagined as a man having two opposite heads or faces, thus symbolizing such opposite concepts as the entrance and the exit, the beginning and the end, the youth and the old age, etc. Casagrande et al. [1,2] gave this name for the first time to glass spherical microparticles whose one hemispheres were prepared hydrophilic and the other ones hydrophobic. The authors named them "Janus beads", studied their properties at oil/water interfaces, and concluded that the Janus beads behaved differently from ordinary solid microparticles. De Gennes [3] cited this work in his Nobel lecture, calling these microparticles "Janus grains", and attracted much attention to this field of research.

Nowdays the term "JPs" denotes nano- and microparticles of different origin, opposite sites of which show opposite or very different chemical and/ or physical properties. JPs may be prepared as substrate particles of various shapes, which are differently treated and/or coated by special substances or agents, or as composite particles consisting of parts of different materials. JPs may be composed of polymers, metals, metal oxides, etc., with forming organic-organic, organic-inorganic, and inorganicinorganic composite particles of various (sometimes rather complex) architectures. The state of the art in this field is reflected in a number of reviews [411], which demonstrate a great interest to JPs over the past two decades. This is due to the potential applications of JPs in solar energy conversion, photocatalysis and environmental remediation [10], various applied fields of science [12-16], textile industry [17], electronics [18], biology and medicine $[11,16,19,20]$.

Both the mechanical behavior and functional properties of JPs are expected to strongly depend on their strained state and stored strain energy, as is the case with other types of composite nanoparticles.

Corresponding author:A.L. Kolesnikova, e-mail: anna.kolesnikova.physics@gmail.com 
(a)

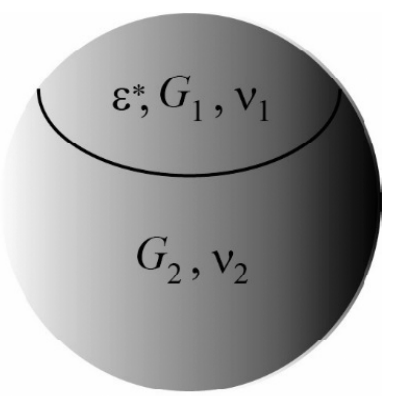

(b)

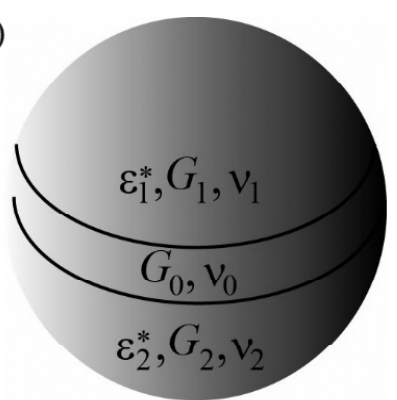

Fig. 1. Janus particle (a) and spherical heterostructure with a quantum well (b). The domains in contact have a mismatch in lattice parameters, which is characterized by dilatation eigenstrains $\varepsilon^{*}, \varepsilon_{1}{ }^{*}, \varepsilon_{2}{ }^{*}$. Here $G_{i}$ and $v_{l}(i=1,2,3)$ are the shear moduli and Poisson ratios, respectively, of the particle domains.

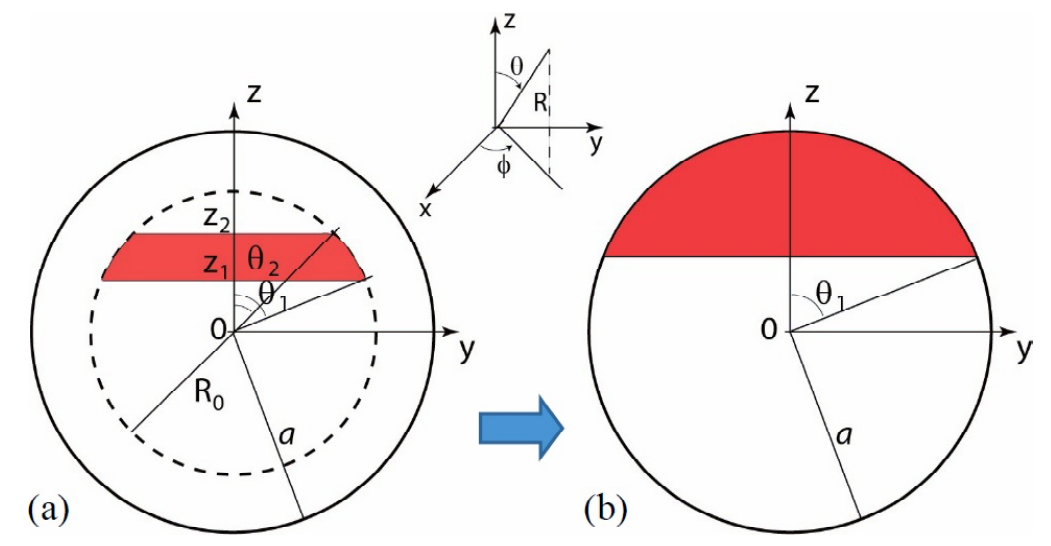

Fig. 2. A geometric scheme for the elastic problem for a Janus particle. (a) Dilatation inclusion in the form of a truncated spherical segment in a spherical particle. (b) Janus particle. The figures show the sectional plane $x=0$ of a spherical particle.

The elastic fields in JPs can be caused by both the external forces, for example, the electrostatic ones, arising due to the distribution of charges of opposite signs on the JP surface, and internal forces caused by the mismatch of the lattice parameters of crystalline materials of different neighboring parts of JPs. To the best of our knowledge, for today there is no analytical solution describing the elastic fields and the strain energy in any kind of JPs.

In the present paper, we confine ourselves to the analysis of the stress-strain state in a spherical JP, two parts of which are separated by a flat interface and have a mismatch in their lattice parameters (Fig. 1a). We calculate analytically the elastic fields and the strain energy in such a JP under the conditions of elastic isotropy of its parts and equality of their shear moduli $G_{1}=G_{2}=G$ and Poisson ratios $v_{1}=v_{2}=v$. It is worth noting that our method of calculations makes it possible to solve similar elastic problems for spherical particles with any number of mismatched regions. In particular, for example, this concerns a spherical heterostructure with a quantum well (Fig. 1b), which is of special interest for optoelectronics.
To solve the elastic problem for such a JP (Fig. 1a), we will use the earlier results obtained for a homogeneously dilatational inclusion in the shape of a truncated sphere embedded in an infinite elastic medium [21].

\section{SPHERICAL CORE-SHELL PARTICLE WITH A TRUNCATED CORE}

Consider a spherical core-shell particle with a truncated core as an elastic sphere of radius $a$ with an axially symmetric truncated spherical inclusion of the geometry determined by spherical coordinates $R_{0}, \theta_{1}$, and $\theta_{2}$ (Fig. 2a). In the Cartesian coordinate system with the origin at the center of the sphere, the inclusion faces normally cross the $z$-axis at the points $z_{1}$ and $z_{2}$. As noted above, we assume that the shear moduli and the Poisson ratios for the sphere and inclusion are the same. Let the inclusion be the subject of $3 \mathrm{D}$ homogeneous dilatational eigenstrain as follows:

$\varepsilon_{x x}^{*}=\varepsilon_{y y}^{*}=\varepsilon_{z z}^{*}=\varepsilon^{*}$. 
The elastic field of an inclusion in the spherical particle can be represented as the sum of the elastic field of the inclusion in an infinite medium and an extra elastic field, which is designed in such a manner that the boundary conditions on the sphere surface are satisfied:

$$
\begin{aligned}
& \boldsymbol{\sigma}={ }^{\infty} \boldsymbol{\sigma}+{ }^{i} \boldsymbol{\sigma}, \\
& \left.\boldsymbol{\sigma} \cdot \mathbf{n}\right|_{s}=0 .
\end{aligned}
$$

Here $\boldsymbol{\sigma}$ is the stress caused by the inclusion in the sphere, ${ }^{\infty} \boldsymbol{\sigma}$ is the stress caused by the inclusion in an infinite medium, ' $\boldsymbol{\sigma}$ is the extra (image) stress; $S$ is the surface of the sphere, and $\mathbf{n}$ is a normal to the sphere surface.

According to Kolesnikova et al. [21], the stress field of a dilatation inclusion in the form of a truncated spherical segment ${ }^{\infty} \boldsymbol{\sigma}$ in a spherical coordinate system $(R, \theta, \varphi)$ with the origin at the sphere center reads: inside inclusion $\left(R \leq R_{0}, z_{1} \leq z \leq z_{2}\right)$

$$
\begin{aligned}
& { }^{\infty} \sigma_{R R}^{i n}=\frac{(1+v) G \varepsilon^{*}}{(1-v)}\left(-2+2 \cos ^{2} \theta+\sum_{n=2}^{\infty} B_{n} n(n-1)\left(\frac{R}{R_{0}}\right)^{n-2} P_{n}(\cos \theta)\right), \\
& { }^{\infty} \sigma_{R \theta}^{i n}=\frac{(1+v) G \varepsilon^{*}}{(1-v)}\left(-2 \sin \theta \cos \theta+\sum_{n=2}^{\infty} B_{n}(n-1)\left(\frac{R}{R_{0}}\right)^{n-2} P_{n}^{1}(\cos \theta)\right), \\
& { }^{\infty} \sigma_{\theta \theta}^{i n}=\frac{(1+v) G \varepsilon}{2(1-v)}\left(-2+2 \sin ^{2} \theta+\sum_{n=2}^{\infty} B_{n}\left(\frac{R}{R_{0}}\right)^{n-2}\left(-n^{2} P_{n}(\cos \theta)-\cot \theta P_{n}^{1}(\cos \theta)\right)\right), \\
& { }^{\infty} \sigma_{\varphi \varphi}^{i n}=\frac{(1+v) G \varepsilon}{(1-v)}\left(-2+\sum_{n=2}^{\infty} B_{n}\left(\frac{R}{R_{0}}\right)^{n-2}\left(n P_{n}(\cos \theta)+\cot \theta P_{n}^{1}(\cos \theta)\right)\right),
\end{aligned}
$$

outside inclusion $\left(R_{0}<R ; R<R_{0}\right.$, and $z \leq z_{1}$ or $\left.z_{2} \leq z\right)$

$$
\begin{aligned}
& { }^{\infty} \sigma_{R R}^{\text {out }}=\frac{(1+v) G \varepsilon}{(1-v)}\left\{\begin{array}{l}
\sum_{n=2}^{\infty} B_{n} n(n-1)\left(\frac{R}{R_{0}}\right)^{n-2} P_{n}(\cos \theta), \quad R<R_{0}, \quad z \leq \mathrm{z}_{1} \text { or } z_{2} \leq \mathrm{z} \\
\sum_{n=0}^{\infty} D_{n}(n+1)(n+2)\left(\frac{R_{0}}{R}\right)^{n+3} P_{n}(\cos \theta), R_{0}<R
\end{array}\right. \\
& \sigma_{R \theta}^{\infty}=\frac{(1+v) G \varepsilon}{(1-v)}\left\{\begin{array}{l}
\sum_{n=2}^{\infty} B_{n}(n-1)\left(\frac{R}{R_{0}}\right)^{n-2} P_{n}^{1}(\cos \theta), \quad R<R_{0}, z \leq z_{1} \text { or } z_{2} \leq z \\
\sum_{n=0}^{\infty}-D_{n}(n+2)\left(\frac{R_{0}}{R}\right)^{n+3} P_{n}^{1}(\cos \theta), R_{0}<R
\end{array},\right. \\
& { }^{\infty} \sigma_{\theta \theta}^{\text {out }}=\frac{(1+v) G \varepsilon^{*}}{(1-v)}\left\{\begin{array}{l}
\sum_{n=2}^{\infty} B_{n}\left(\frac{R}{R_{0}}\right)^{n-2}\left(-n^{2} P_{n}(\cos \theta)-\cot \theta P_{n}^{1}(\cos \theta)\right), \quad R<R_{0}, \quad z \leq z_{1} \text { or } z_{2} \leq z \\
\sum_{n=0}^{\infty} D_{n}\left(\frac{R_{0}}{R}\right)^{n+3}\left(-(n+1)^{2} P_{n}(\cos \theta)-\cot \theta P_{n}^{1}(\cos \theta)\right), \quad R_{0}<R
\end{array},\right. \\
& { }^{\infty} \sigma_{\varphi \varphi}^{\text {out }}=\frac{(1+v) G \varepsilon}{(1-v)}\left\{\begin{array}{l}
\sum_{n=2}^{\infty} B_{n}\left(\frac{R}{R_{0}}\right)^{n-2}\left(n P_{n}(\cos \theta)+\cot \theta P_{n}^{1}(\cos \theta)\right), \quad R<R_{0}, \quad z \leq z_{1} \text { or } z_{2} \leq z \\
\sum_{n=0}^{\infty} D_{n}\left(\frac{R_{0}}{R}\right)^{n+3}\left(-(n+1) P_{n}(\cos \theta)+\cot \theta P_{n}^{1}(\cos \theta)\right), \quad R_{0}<R
\end{array}\right.
\end{aligned}
$$


where

$$
\begin{aligned}
& B_{n}=\left.\frac{1}{(2 n-1)}\left(\frac{1}{(2 n+1)} P_{n+1}\left(t_{0}\right)-\frac{2(2 n-1)}{(2 n+1)(2 n-3)} P_{n-1}\left(t_{0}\right)+\frac{1}{(2 n-3)} P_{n-3}\left(t_{0}\right)\right)\right|_{\cos \theta_{1}} ^{\cos \theta_{2}} \\
& D_{n}=\left.\frac{1}{(2 n+3)}\left(\frac{1}{(2 n+5)} P_{n+3}\left(t_{0}\right)-\frac{2(2 n+3)}{(2 n+5)(2 n+1)} P_{n+1}\left(t_{0}\right)+\frac{1}{(2 n+1)} P_{n-1}\left(t_{0}\right)\right)\right|_{\cos \theta_{1}} ^{\cos \theta_{2}}
\end{aligned}
$$

$P_{n}(\cos \theta)$ and $P_{n}^{1}(\cos \theta)$ are the Legendre polynomials and the associated Legendre polynomials, respectively [22]. In formulas (4) and (5), the Lurie designations for the coefficients of the series $B_{n}$ and $D_{n}$ are adopted [23].

The extra stress field ' $\boldsymbol{\sigma}$ is written in the form of the Lurie series [23] corresponding to the internal problem of an elastic sphere:

$$
\begin{aligned}
& { }^{i} \sigma_{R R}=2 G \sum_{n=0}^{\infty}\left({ }^{i} A_{n}(n+1)\left(n^{2}-n-2-2 v\right)\left(\frac{R}{a}\right)^{n}+{ }^{i} B_{n} n(n-1)\left(\frac{R}{a}\right)^{n-2}\right) P_{n}(\cos \theta), \\
& { }^{i} \sigma_{R \theta}=2 G \sum_{n=1}^{\infty}\left({ }^{i} A_{n}\left(n^{2}+2 n-1+2 v\right)\left(\frac{R}{a}\right)^{n}+{ }^{i} B_{n}(n-1)\left(\frac{R}{a}\right)^{n-2}\right) P_{n}^{1}(\cos \theta), \\
& { }^{i} \sigma_{\theta \theta}=2 G\left[-\sum_{n=0}^{\infty}\left({ }^{i} A_{n}(n+1)\left(n^{2}+4 n+2+2 v\right)\left(\frac{R}{a}\right)^{n}+{ }^{i} B_{n} n^{2}\left(\frac{R}{a}\right)^{n-2}\right) P_{n}(\cos \theta)-\right. \\
& \left.\sum_{n=1}^{\infty}\left({ }^{i} A_{n}(n+5-4 v)\left(\frac{R}{a}\right)^{n}+{ }^{i} B_{n}\left(\frac{R}{a}\right)^{n-2}\right) \cot \theta P_{n}^{1}(\cos \theta)\right], \\
& { }^{i} \sigma_{\varphi \varphi}=2 G\left[\sum_{n=0}^{\infty}\left({ }^{i} A_{n}(n+1)(n-2-2 v-4 n v)\left(\frac{R}{a}\right)^{n}+{ }^{i} B_{n} n\left(\frac{R}{a}\right)^{n-2}\right) P_{n}(\cos \theta)+\right. \\
& \left.\sum_{n=1}^{\infty}\left({ }^{i} A_{n}(n+5-4 v)\left(\frac{R}{a}\right)^{n}+{ }^{i} B_{n}\left(\frac{R}{a}\right)^{n-2}\right) \cot \theta P_{n}^{1}(\cos \theta)\right],
\end{aligned}
$$

where ${ }^{i} A_{n}$ and ${ }^{\prime} B_{n}$ are unknown coefficients which are determined from the boundary conditions of the problem.

To find the coefficients ${ }^{i} A_{n}$ and ${ }^{i} B_{n}$, we represent boundary equations (3) in the componential form:

$$
\begin{aligned}
& \left.{ }^{\infty} \sigma_{R R}^{\text {out }}\right|_{R=a}+\left.{ }^{i} \sigma_{R R}\right|_{R=a}=0 ; \\
& \left.{ }^{\infty} \sigma_{R \theta}^{\text {out }}\right|_{R=a}+\left.{ }^{i} \sigma_{R \theta}\right|_{R=a}=0 .
\end{aligned}
$$

Here we suppose that the inclusion is completely located in the sphere, therefore its stresses with the index "out" are included in the boundary conditions.

Due to the orthogonality of the terms of the Lurie series, from the system of equations (7), we obtain the following system of equations for the coefficients ${ }^{\prime} A_{n}$ and ${ }^{\prime} B_{n}(n=2,3 \ldots)$ :

$$
\begin{aligned}
& \frac{(1+v) \varepsilon^{*}}{(1-v)} D_{n}(n+1)(n+2)\left(\frac{R_{0}}{a}\right)^{n+3}+2^{i} A_{n}(n+1)\left(n^{2}-n-2-2 v\right)+2^{i} B_{n} n(n-1)=0, \\
& -\frac{(1+v) \varepsilon}{(1-v)} D_{n}(n+2)\left(\frac{R_{0}}{a}\right)^{n+3}+2^{i} A_{n}\left(n^{2}+2 n-1+2 v\right)+2^{i} B_{n}(n-1)=0 .
\end{aligned}
$$


The solution of system ( 8 ) is given by

$$
\begin{aligned}
& { }^{i} A_{n}=\frac{\varepsilon^{*}(1+v)}{4(1-v)} \frac{(n+2)(2 n+1)}{\left(n^{2}+n+1+v+2 v n\right)} D_{n}\left(\frac{R_{0}}{a}\right)^{n+3},(n=2,3, \ldots) \\
& { }^{i} B_{n}=-\frac{\varepsilon^{*}(1+v)}{4(1-v)} \frac{(n+1)(n+2)(2 n+3)}{\left(n^{2}+n+1+v+2 v n\right)} D_{n}\left(\frac{R_{0}}{a}\right)^{n+3},(n=2,3, \ldots)
\end{aligned}
$$

The coefficient ${ }^{i} A_{0}$ is calculated from Eq. (8.1) at $n=0$. We suppose that ${ }^{i} B_{0}=0$, because this coefficient does not contribute to any of the series that represent the components of displacements or stresses [23]. As a result, for 0 -coefficients, we obtain:

$$
\begin{aligned}
& { }^{i} A_{0}=\frac{\varepsilon}{2(1-v)} D_{0}\left(\frac{R_{0}}{a}\right)^{3}, \\
& { }^{i} B_{0}=0 .
\end{aligned}
$$

The coefficient ${ }^{~} A_{1}$ is calculated from one of Eqs. (8) at $n=1$. Taking into account the fact that the coefficient ${ }^{i} B_{1}$ is responsible for the rigid displacement of the body as a whole [23] and therefore does not contribute to stresses, we take it to be zero. Then the coefficients ${ }^{\prime} A_{1}$ and ${ }^{i} B_{1}$ are:

$$
\begin{aligned}
& { }^{i} A_{1}=\frac{3 \varepsilon}{4(1-v)} D_{1}\left(\frac{R_{0}}{a}\right)^{4}, \\
& { }^{i} B_{1}=0 .
\end{aligned}
$$

Thus, we have found the stresses generated by the inclusion in a sphere (Fig. 2a), see Eqs. (2), (4)-(6) with (9)-(11). This means that the elastic problem for a truncated spherical inclusion in a spherical particle (Fig. 2a) has been solved.

Our analysis of the found solution as well as the plotted distributions of stress components $\sigma_{R R}$ and $\sigma_{R \theta}$ over the sphere surface (not shown here) have demonstrated the satisfaction of boundary conditions (7.1) and (7.2).

In Figs. 3a, 3c, 3e, and 3g, the maps of stress components of a hemispherical dilatation inclusion in an elastic sphere, calculated numerically for its central meridional section, are shown in a cylindrical coordinate system $(r, \varphi, z)$ with the origin at the sphere center. For comparison, in Figs. $3 b, 3 d, 3 f$, and $3 \mathrm{~h}$ similar stress components of this inclusion in an infinite medium are represented.

Based on the displacement field of a truncated spherical inclusion in an infinite medium ${ }^{\circ} u_{R}[21]$ and the general form of displacements in axisymmetric elastic problems for a sphere ${ }^{\prime} u_{R}$ [23], with using the coefficients ${ }^{\prime} A_{n}$ and ${ }^{\prime} B_{n}(n=0,1,2, \ldots)$ figuring in Eqs. (9)-(11), we can write now the nonzero displacement components for our case (Fig. 2a):

$u_{R}={ }^{\infty} u_{R}+{ }^{i} u_{R}$,

$$
u_{\theta}={ }^{\infty} u_{\theta}+{ }^{i} u_{\theta} .
$$

The terms ${ }^{\infty} u_{R}$ and ${ }^{\infty} u_{\theta}$ have the form [21]: inside inclusion $\left(R<R_{0}, z_{1} \leq Z \leq Z_{2}\right)$

$$
{ }^{\infty} u_{R}^{i n}=\frac{(1+v) \varepsilon R_{0}}{2(1-v)}\left(\cos \theta\left[\frac{2 R}{R_{0}} \cos \theta-\left(\cos \theta_{2}+\cos \theta_{1}\right)+\frac{1}{2}\left(\cos ^{2} \theta_{2}-\cos ^{2} \theta_{1}\right)\right]+\sum_{n=2}^{\infty} B_{n} n\left(\frac{R}{R_{0}}\right)^{n-1} P_{n}(\cos \theta)\right),
$$




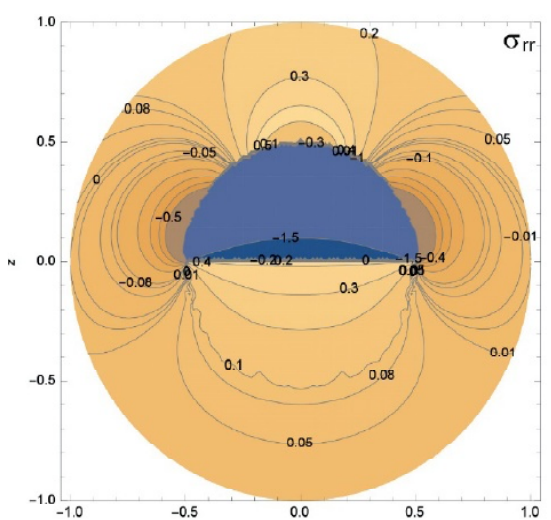

(a)

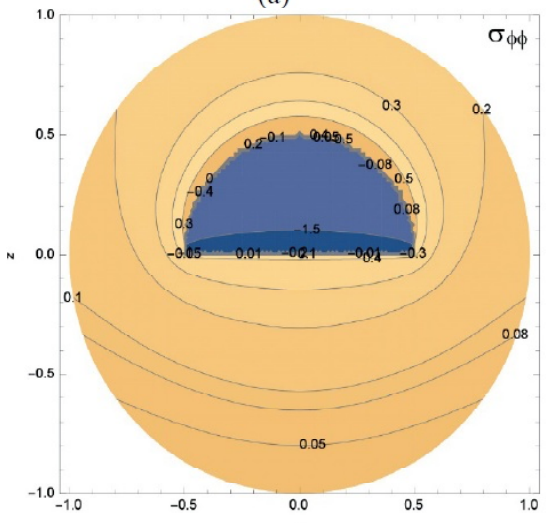

(c)

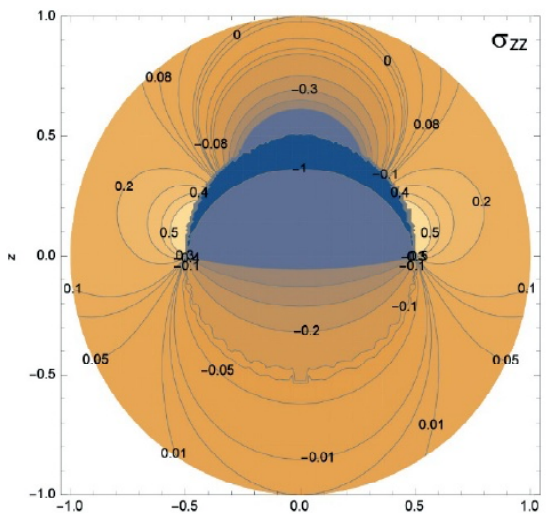

(e)

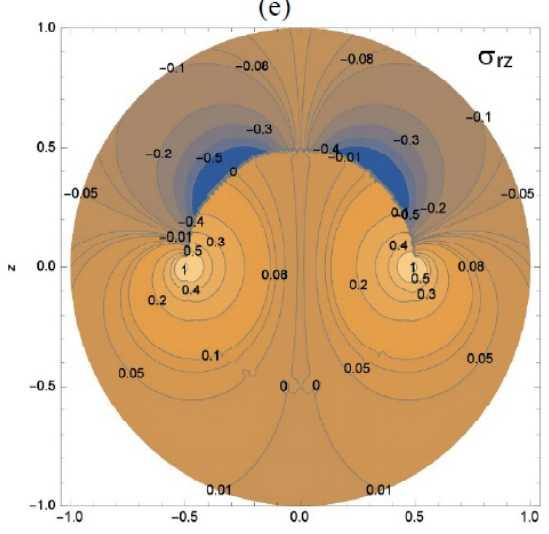

(g)

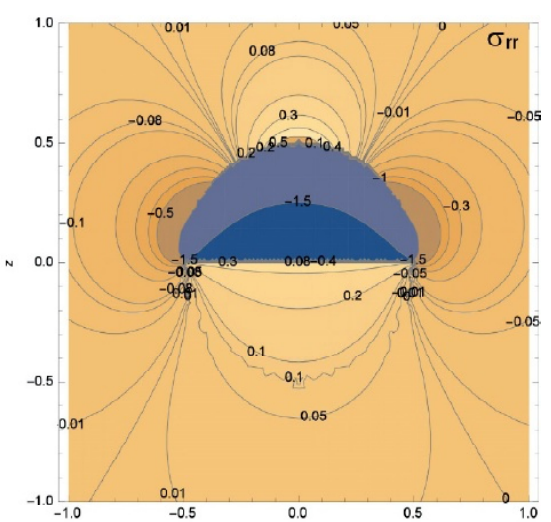

(b)

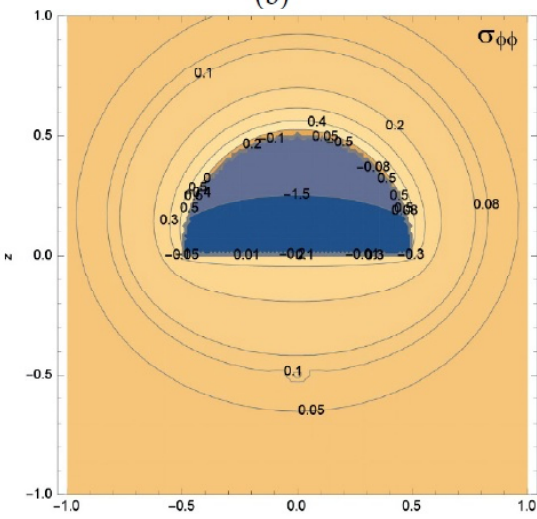

(d)

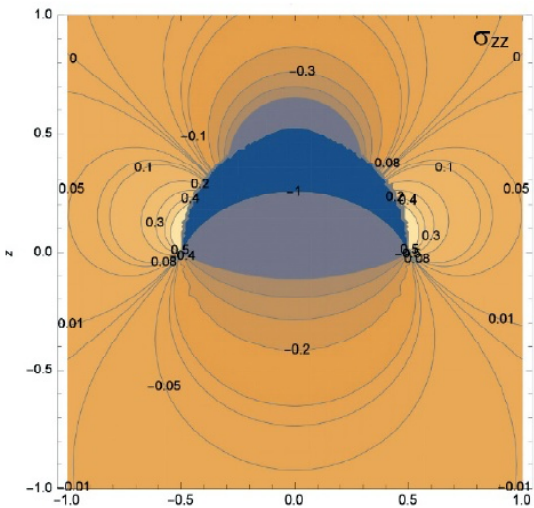

(f)

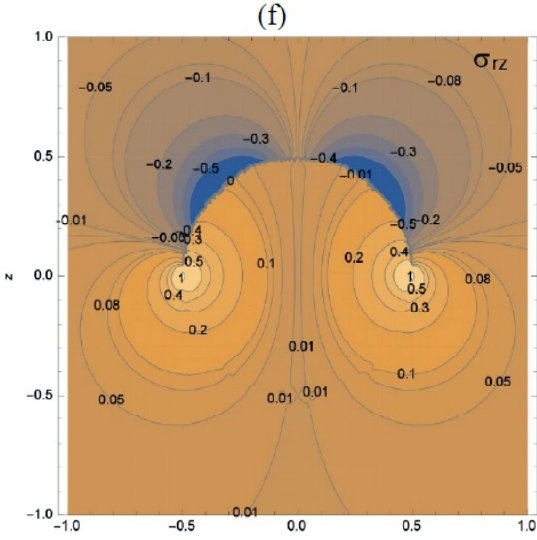

(h)

Fig. 3. Maps of stresses caused by a hemispherical dilatation inclusion in an elastic sphere $(a, c, e, g)$ and an infinite elastic medium $(b, d, f, h)$. The stress values are given in units of $G \varepsilon^{*}(1+v) /(1-v)$. The map coordinates are normalized to the sphere radius $a$. The calculations are made for the material with $v=0.3$. 


$$
\begin{aligned}
& { }^{\infty} u_{\theta}^{i n}=\frac{(1+v) \varepsilon^{*} R_{0}}{2(1-v)}\left(-\sin \theta\left[\frac{2 R}{R_{0}} \cos \theta-\left(\cos \theta_{2}+\cos \theta_{1}\right)+\frac{1}{2}\left(\cos ^{2} \theta_{2}-\cos ^{2} \theta_{1}\right)\right]+\right. \\
& \left.\sum_{n=2}^{\infty} B_{n}\left(\frac{R}{R_{0}}\right)^{n-1} P_{n}^{1}(\cos \theta)\right)
\end{aligned}
$$

outside inclusion $\left(R<R_{0}, z>z_{2} ; R<R_{0}, Z<z_{1} ; R_{0}<R\right)$ )

$$
{ }^{\infty} u_{R}^{\text {out }}=\frac{(1+v) \varepsilon^{*} R_{0}}{2(1-v)}\left\{\begin{array}{l}
\cos \theta\left\{\begin{array}{l}
\left(\cos \theta_{2}-\cos \theta_{1}\right), z>z_{2} \\
\left(\cos \theta_{1}-\cos \theta_{2}\right), z<z_{1}
\end{array}\right\}+\frac{1}{2}\left(\cos ^{2} \theta_{2}-\cos ^{2} \theta_{1}\right) \cos \theta+ \\
\sum_{n=2}^{\infty} B_{n} n\left(\frac{R}{R_{0}}\right)^{n-1} P_{n}(\cos \theta), R<R_{0} \\
\sum_{n=0}^{\infty}-D_{n}(n+1)\left(\frac{R_{0}}{R}\right)^{n+2} P_{n}(\cos \theta), R_{0}<R
\end{array},\right.
$$

$$
{ }^{\infty} u_{\theta}^{\text {out }}=\frac{(1+v) \varepsilon R_{0}}{2(1-v)}\left\{\begin{array}{l}
-\sin \theta\left\{\begin{array}{l}
\left(\cos \theta_{2}-\cos \theta_{1}\right), z>z_{2} \\
\left(\cos \theta_{1}-\cos \theta_{2}\right), z<z_{1}
\end{array}\right\}-\frac{1}{2}\left(\cos ^{2} \theta_{2}-\cos ^{2} \theta_{1}\right) \sin \theta+ \\
\sum_{n=2}^{\infty} B_{n}\left(\frac{R}{R_{0}}\right)^{n-1} P_{n}^{1}(\cos \theta), R<R_{0} \\
\sum_{n=1}^{\infty} D_{n}\left(\frac{R_{0}}{R}\right)^{n+2} P_{n}^{1}(\cos \theta), R_{0}<R
\end{array},,\right.
$$

${ }^{\infty} u_{\varphi}^{\text {out }}=0$.

The extra terms are

$$
\begin{aligned}
& { }^{i} u_{R}=a \sum_{n=0}^{\infty}\left({ }^{i} A_{n}(n+1)(n-2+4 v)\left(\frac{R}{a}\right)^{n+1}+{ }^{i} B_{n} n\left(\frac{R}{a}\right)^{n-1}\right) P_{n}(\cos \theta), \\
& { }^{i} u_{\theta}=a \sum_{n=1}^{\infty}\left({ }^{i} A_{n}(n+5-4 v)\left(\frac{R}{a}\right)^{n+1}+{ }^{i} B_{n}\left(\frac{R}{a}\right)^{n-1}\right) P_{n}^{1}(\cos \theta) .
\end{aligned}
$$

Similarly, to the fields of stresses and displacements, one can found the formula for the elastic dilatation $\Delta[21,23]:$

$$
\Delta=\underbrace{\left\{\begin{array}{c}
\frac{2(2 v-1) \varepsilon^{*}}{(1-v)}, \text { inside inclusion } \\
0, \text { outside inclusion }
\end{array}\right.}_{\infty_{\Delta}}\}+\underbrace{2(2 v-1) \sum_{n=0}^{\infty}{ }^{i} A_{n}(n+1)(2 n+3)\left(\frac{R}{a}\right)^{n} P_{n}(\cos \theta)}_{\text {' }_{\Delta}} .
$$

Formula (16) shows that a dilatation inclusion, which, regardless of its form, does not create any elastic dilatation in the surrounding infinite medium [21,24], creates it in the sphere of finite radius. Moreover, the constancy of dilatation within the inclusion is violated, see Eq. (16). Both these conclusions are illustrated by Fig. 4, where a map of the elastic dilatation inside and outside the hemispherical inclusion embedded in an elastic sphere is shown in the meridional section of the sphere.

The strain energy caused by the hemispherical inclusion in the sphere is calculated as the work spent to generate the inclusion in its own stress field in the sphere [24] as follows: 


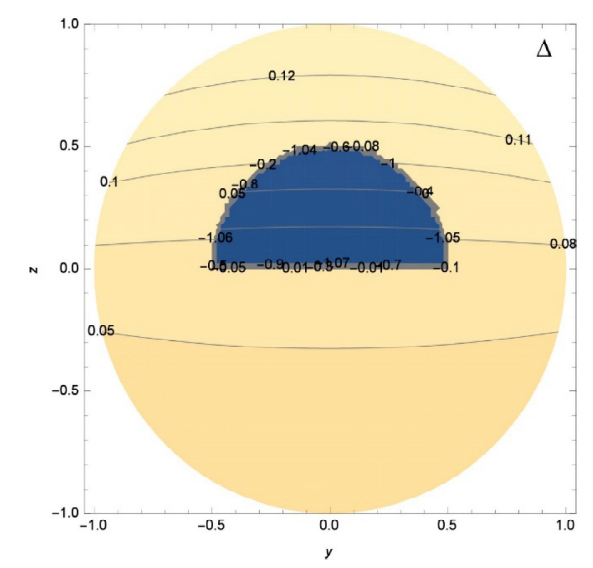

Fig. 4. Map of elastic dilatation caused by the hemispherical inclusion in the sphere. The dilatation values are given in units of $\varepsilon^{*}$. The map coordinates are normalized to the sphere radius $a$. Calculations are made for the material with $v=0.3$.

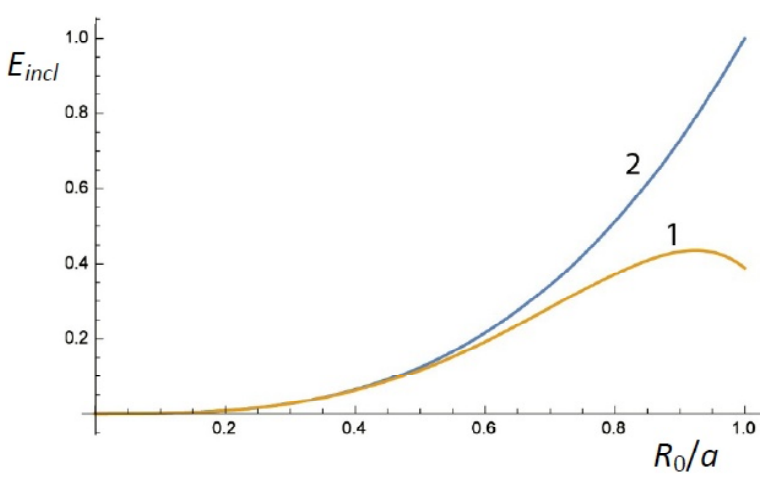

Fig. 5. The strain energy of a hemispherical dilatation inclusion $E_{\text {incl }}$ as a function of its normalized radius $R_{0} / a$ in a sphere of finite radius a (curve 1 ) and in an infinite medium (curve 2 ). The energy values are given in units of $4 \pi(1+v) G \varepsilon^{* 2} a^{3} /(3(1-v))$ for $v=0.3$.

$$
\begin{aligned}
& E_{\text {incl }}=-\frac{1}{2} \int_{V} \sigma_{i j} \varepsilon_{i j}^{*} d V=-\frac{1}{2} \int_{V_{\text {ned }}}\left({ }^{\infty} \sigma_{R R}^{i n}+{ }^{\infty} \sigma_{\theta \theta}^{i n}+{ }^{\infty} \sigma_{\varphi \varphi}^{\text {in }}\right) \varepsilon \dot{d} d V- \\
& \frac{1}{2} \int_{V_{\text {nod }}}\left({ }^{i} \sigma_{R R}+{ }^{i} \sigma_{\theta \theta}+{ }^{i} \sigma_{\varphi \varphi}\right) \varepsilon d V={ }^{\infty} E_{i n c l}+{ }^{i} E_{\text {incl }}= \\
& \frac{2(1+v) G \varepsilon^{* 2}}{(1-v)} V_{\text {incl }}+2 G(1+v) \varepsilon \int_{v_{\text {nod }}} \sum_{n=0}^{\infty}{ }^{i} A_{n}(n+1)(2 n+3)\left(\frac{R}{a}\right)^{n} P_{n}(\cos \theta) \mathrm{d} V= \\
& \frac{2(1+v) G \varepsilon^{2}}{(1-v)} V_{\text {incl }}+\frac{(1+v) G \varepsilon^{2}}{2(1-v)} \int_{V_{\text {net }}} \sum_{n=0}^{\infty} \frac{(n+1)(n+2)(2 n+1)(2 n+3)}{\left(n^{2}+n+1+v+2 v n\right)} D_{n}\left(\frac{R_{0}}{a}\right)^{n+3}\left(\frac{R}{a}\right)^{n} P_{n}(\cos \theta) \mathrm{d} V= \\
& \frac{2(1+v) G \varepsilon^{2}}{(1-v)} V_{\text {incl }}+\frac{(1+v) G \varepsilon^{2}}{2(1-v)} \sum_{n=0}^{\infty} \frac{(n+1)(n+2)(2 n+1)(2 n+3)}{\left(n^{2}+n+1+v+2 v n\right)}\left(\frac{R_{0}}{a}\right)^{n+3} \times \\
& D_{n} \int_{\theta_{2}}^{\theta_{1}} \int_{0}^{R_{0}} \int_{0}^{2 \pi}\left(\frac{R}{a}\right)^{n} P_{n}(\cos \theta) \sin \theta R^{2} \mathrm{~d} R \mathrm{~d} \theta \mathrm{d} \varphi=\frac{2(1+v) G \varepsilon^{* 2}}{(1-v)} V_{\text {incl }}+ \\
& \frac{2 \pi(1+v) G \varepsilon^{2}}{(1-v)} a^{3}\left(\frac{R_{0}}{a}\right)^{6} D_{0}\left(\cos \theta_{2}-\cos \theta_{1}\right)+\frac{(1+v)^{2} G \varepsilon^{2}}{(1-v)} \pi a^{3} \times \\
& \sum_{n=1}^{\infty} \frac{(n+2)(2 n+1)(2 n+3)}{n(n+3)\left(n^{2}+n+1+v+2 v n\right)}\left(\frac{R_{0}}{a}\right)^{2(n+3)} D_{n}\left(\sin \theta_{2} P_{n}^{1}\left(\cos \theta_{2}\right)-\sin \theta_{1} P_{n}^{1}\left(\cos \theta_{1}\right)\right)= \\
& \frac{2(1+v) G \varepsilon^{2}}{(1-v)} V_{\text {incl }}\left\{1-\frac{1}{4}\left(\frac{R_{0}}{a}\right)^{3}\left(3\left(\cos \theta_{2}-\cos \theta_{1}\right)-\left(\cos ^{3} \theta_{2}-\cos ^{3} \theta_{1}\right)\right)+\right. \\
& \left.\frac{3(1+v)}{4\left(\cos \theta_{2}-\cos \theta_{1}\right)} \sum_{n=1}^{\infty} \frac{(n+2)(2 n+1)(2 n+3)}{n(n+3)\left(n^{2}+n+1+v+2 v n\right)}\left(\frac{R_{0}}{a}\right)^{2 n+3} D_{n}\left(\sin \theta_{2} P_{n}^{1}\left(\cos \theta_{2}\right)-\sin \theta_{1} P_{n}^{1}\left(\cos \theta_{1}\right)\right)\right\} \text {. }
\end{aligned}
$$

Here the term ${ }^{\infty} E_{\text {incl }}=2(1+v) G \varepsilon^{2} V_{\text {ind }} /(1-v)$ is the strain energy of the dilatation inclusion in an infinite elastic medium [24], $V_{\text {incl }}=2 \pi R_{0}^{3}\left(\cos \theta_{2}-\cos \theta_{1}\right) / 3$ is the volume of a truncated spherical inclusion (Fig. 2a), ${ }^{i} E_{\text {incl }}$ is an extra strain energy term which arises due to the finiteness of the sphere radius,

$$
D_{0}=\left.\frac{1}{3}\left(\frac{1}{5} P_{3}\left(t_{0}\right)-\frac{6}{5} P_{1}\left(t_{0}\right)+P_{-1}\left(t_{0}\right)\right)\right|_{\cos \theta_{1}} ^{\cos \theta_{2}}=-\frac{1}{6}\left(3\left(\cos \theta_{2}-\cos \theta_{1}\right)-\left(\cos ^{3} \theta_{2}-\cos ^{3} \theta_{1}\right)\right),
$$




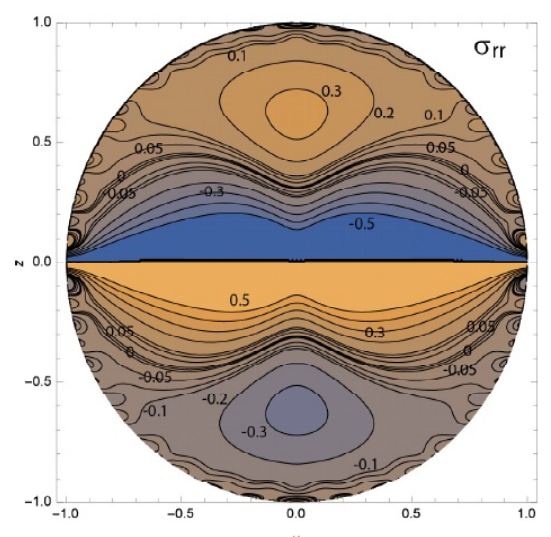

(a)

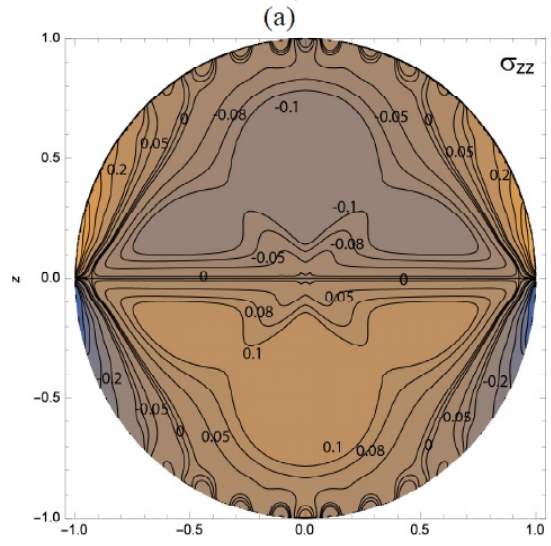

(c)

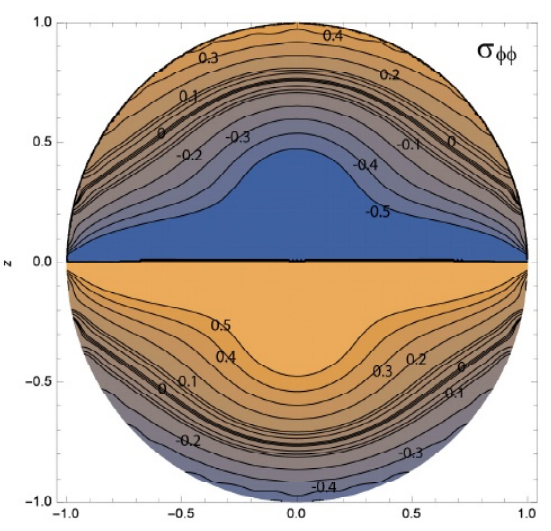

(b)

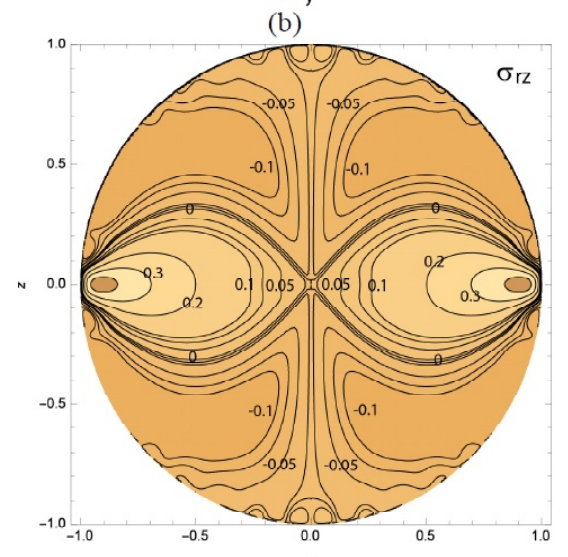

(d)

Fig. 6. Maps of the cylindrical stress components in the JP, consisting of two equal hemispheres with equatorial position $(z=0)$ of the interface. The stress values are given in units of $G \varepsilon^{*}(1+v) /(1-v)$, where the eigenstrain $\varepsilon^{*}$ is attributed to the upper hemisphere. The map coordinates are normalized to the JP radius a. The calculations are made for the material with $v=0.3$.

and $D_{n}$ is determined in the comments to Eq. (5). It is worth noting that in an infinite medium, the strain energy of a dilatation inclusion does not depend on its shape $[21,24]$.

In Fig. 5, the dependence of the strain energy of a hemispherical dilatation inclusion (with $\theta_{1}=\pi / 2$ and $\theta_{2}=0$ in Eq. (17)) on its radius $R_{0}$ is shown for both the cases of finite-radius (curve 1) and infinite-radius (curve 2) spheres. It is seen that the influence of the free surface of the sphere on the inclusion strain energy becomes noticeable, when the inclusion radius $R_{0}$ reaches a half of the sphere radius $a$.

Our analysis has shown that the series in Eq. (17) poorly converge when $\left(R_{0} / a\right) \rightarrow 1$, therefore, the curve 1 in Fig. 5 is considered conditionally correct at $0.9 \leq\left(R_{0} / a\right) \leq 1$. Nevertheless, it seems reasonable that in the sphere the strain energy attains its maximum value $E_{\text {incl }}^{\max } \approx 0.44(4 \pi / 3) G(1+v) \varepsilon^{* 2} a^{3} /(1-v) \approx$ $3.42 G \varepsilon^{2} a^{3}$ for $v=0.3$ when $R_{0} / a \approx 0.925$, as it follows from the behavior of this curve.

\section{ELASTIC FIELDS AND STRAIN ENERGY OF JANUS PARTICLE}

From the inclusion shown in Fig. 2a, we proceed to the Janus particle in Fig. 2b. In doing so, we put $R_{0}=a$ and $\theta_{2}=0$ in Eqs. (4), (5), and (9)-(16). Thus, in this case, the JP under cosideration consists of two regions with a mismatch in parameters of their crystal lattices, and the aforementioned dilatational eigenstrain $\varepsilon^{*}$ plays now the role of the misfit parameter for these regions.

The cylindrical stress components and elastic dilatation in the JP, consisting of two equal hemispheres with equatorial position of the interface (Fig. $2 b$ ), are illustrated by stress and dilatation maps in Figs. 6 and 7, respectively, which are built for a meridional section of the JP.

Using Eq. (17) which gives the strain energy of a composite particle with truncated core, we derive at $R_{0}=a$ and $\theta_{2}=0$ the following expression for the strain energy of a JP which consists, in the general case, of two segments of unequal sizes: 


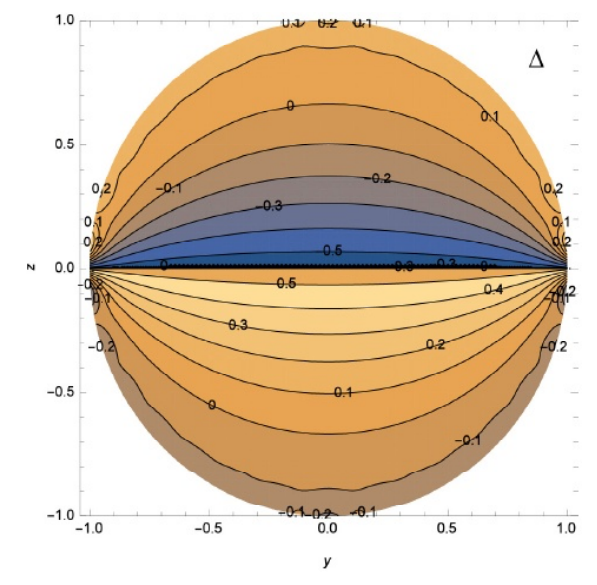

Fig. 7. Map of the elastic dilatation in the JP, consisting of two equal hemispheres with equatorial position $(z=0)$ of the interface. The dilatation values are given in units of the eigenstrain $\varepsilon^{*}$, which is attributed to the upper hemisphere. The map coordinates are normalized to the JP radius $a$. The calculations are made for the material with $v=0.3$.

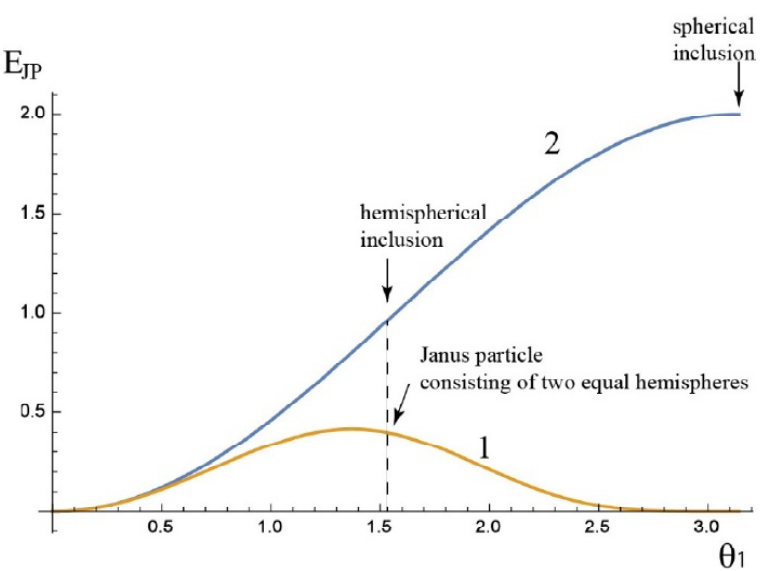

Fig. 8. Strain energies of a JP (curve 1) and a truncated dilatation inclusion embedded in an infinite elastic medium (curve 2) as functions of the interface coordinate $\theta_{1}$. The energy values are given in units of $4 \pi(1+v) G \varepsilon^{* 2} a^{3} /(3(1-v))$. The calculations are made for the material with $v=0.3$.

$$
\begin{aligned}
& E_{J P}=\frac{4 \pi(1+v) G \varepsilon^{2}}{3(1-v)} a^{3}\left(1-\cos \theta_{1}\right)\left\{1-\frac{1}{4}\left(2-3 \cos \theta_{1}+\cos ^{3} \theta_{1}\right)-\right. \\
& \left.\frac{3(1+v)}{4\left(1-\cos \theta_{1}\right)} \sum_{n=1}^{\infty} \frac{(n+2)(2 n+1)(2 n+3)}{n(n+3)\left(n^{2}+n+1+v+2 v n\right)} D_{n} \sin \theta_{1} P_{n}^{1}\left(\cos \theta_{1}\right)\right\} .
\end{aligned}
$$

In deriving Eq. (18), the expression for the inclusion volume has been used. The first term in the curly brackets in Eq. (18) is the strain energy of the dilatation inclusion in the form of a segment, constituting the $\mathrm{JP}$, in an infinite elastic medium.

If the JP consists of two equal hemispheres with the misfit parameter $\varepsilon^{*}$ (at $\theta_{1}=\pi / 2$ in Fig. 2b), Eq. (18) transforms in the following simple formula for the strain energy of the JP:

$$
E_{J P}^{h s}=\frac{2 \pi(1+v) G \varepsilon^{2}}{3(1-v)} a^{3}\left\{1-\frac{3(1+v)}{2} \sum_{n=1}^{\infty} \frac{(n+2)(2 n+1)(2 n+3)}{n(n+3)\left(n^{2}+n+1+v+2 v n\right)} D_{n} P_{n}^{1}(0)\right\},
$$

where

$$
D_{n}=\left.\frac{1}{(2 n+3)}\left(\frac{1}{(2 n+5)} P_{n+3}\left(t_{0}\right)-\frac{2(2 n+3)}{(2 n+5)(2 n+1)} P_{n+1}\left(t_{0}\right)+\frac{1}{(2 n+1)} P_{n-1}\left(t_{0}\right)\right)\right|_{t_{0}=0} ^{t_{0}=1} .
$$

In Fig. 8, the dependence of the strain energy of the JP on the $\theta_{1}$ coordinate of the interface is shown by curve 1. Curve 2 in Fig. 8 shows the similar dependence for a truncated inclusion with the same eigenstrain $\varepsilon^{*}$ embedded in an infinite elastic medium.

As is seen, curve 1 reaches its maximum point which corresponds (for $v=0.3$ ) to the energy value $E_{J P}^{\max } \approx 0.42(4 \pi / 3) G(1+v) \varepsilon^{* 2} a^{3} /(1-v) \approx 3.26 G \varepsilon^{* 2} a^{3}$ which is just a bit smaller than the maximum energy value $E_{\text {incl }}^{\max } \approx 0.44(4 \pi / 3) G(1+v) \varepsilon^{2} a^{3} /(1-v) \approx 3.42 G \varepsilon^{* 2} a^{3}$ of a hemispherical dilatation inclusion in an elastic sphere (see Fig. 5). The slight asymmetry of curve 1 in Fig. 8 near the point $\theta_{1}=\pi / 2$ is apparently associated with the inaccuracy of the calculation of the series (18) and (19) due to their weak convergence.

\section{CONCLUSIONS}

The elastic problem of a spherical core-shell particle with a truncated spherical core has been solved. The elastic fields and strain energy of such a composite particle have been found analytically and analyzed 
numerically in detail. As a special case of this problem, an elastic model for a JP consisting of two, in the general case, unequal spherical segments of crystalline materials with lattice mismatch, has been analyzed as well. The elastic fields and strain energy of such a JP have been calculated and depicted.

It is shown that the free surface strongly affect upon the strain energies both the spherical coreshell particle with a truncated spherical core and JP. In the first case, the screening effect of the free surface becomes significant when the core radius is larger than roughly one half of the sphere radius. In the second case, it is so when the $\theta_{1}$ coordinate of the interface prevails over roughly one-half of radian that is roughly $30^{\circ}$. Interestingly, the maximum strain energies are approximately the same for both the cases in spite of some difference in their geometries.

\section{ACKNOWLEDGEMENTS}

A.L.K., M.Yu.G., M.V.D., and E.C.A. are grateful the grant of the Ministry of Education and Science of the Russian Federation (Decree No. 220) to Togliatti State University under the contract No 14.Z50.31.0039.

S.A.K. and A.M.S. thank the Ministry of Education and Science of the Russian Federation for its financial support (Project No 3.3194.2017/4.6).

A.E.R. acknowledges the grant No 18-02-400006 of the Russian Foundation for Basic Research of the Russian Federation

\section{REFERENCES}

[1] C. Casagrande and M. Veyssié // C. R. Acad. Sci. (Paris) 306 (1988) 1423.

[2] C. Casagrande, P. Fabre, M. Veyssié and E. Raphael // Europhysics Letters 9(3) (1989) 251.

[3] P.G. de Gennes // Science 256(5056) (1992) 495.

[4] A. Walther and A.H.E. Müller // Soft Matter 4 (2008) 663.

[5] F. Wurm and A.F.M. Kilbinger // Angew. Chem. Int. Ed. 48 (2009) 8412.

[6] E. Duguet, A. Désert, A. Perro and S. Ravaine // Chem. Soc. Rev. 40 (2011) 941.
[7] M. Lattuada and T.A. Hatton // Nano Today 6(3) (2011) 286.

[8] A. Walther and A. Müller // Chemical Reviews 113(7) (2013) 5194.

[9] Soft, Hard, and Hybrid Janus Structures: Synthesis, Self-Assembly, and Applications, ed. by Z. Lin and B. Li (World Scientific, New Jersey, USA, 2017).

[10] X. Liu, J. locozzia, Y. Wang, X. Cui, Y. Chen, S. Zhao, Z. Li and Z. Lin // Energy Environ. Sci. 10 (2017) 402.

[11] S. Khoee, A. Nouri, In: Design and Development of New Nanocarriers, Ch. 4, ed. by A.M. Grumezescu (William Andrew Applied Science Publishers, 2018), p. 145.

[12] B.P. Binks and P.D.I. Fletcher // Langmuir 17(16) (2001) 4708.

[13] L-P. Xu, S. Pradhan and S. Chen // Langmuir 23(16) (2007) 8544.

[14] A. Walther, K. Matussek and A.H.E. Müller // ACS Nano 2(6) (2008) 1167.

[15] M. Yoshida, K-H. Roh, S. Mandal, S. Bhaskar, D. Lim, H. Nandivada, X. Deng and J. Lahann // Advanced Materials 21(48) (2009) 4920.

[16] G.A. Sotiriou, A.M. Hirt, P-Y. Lozach, A. Teleki, F. Krumeich and S.E. Pratsinis // Chem. Mater. 23(7) (2011) 1985.

[17] A. Synytska, R. Khanum, L. Ionov, Ch. Cherif and C. Bellmann // ACS Appl. Mater. Interfaces 3(4) (2011) 1216.

[18] T. Nisisako, T. Torii, T. Takahashi and Y. Takizawa // Adv. Mater. 18(9) (2006) 1152.

[19] Sh-H. Hu and X. Gao // J. Am. Chem. Soc. 132(21) (2010) 7234.

[20] L.Y. Wu, B.M. Ross, S.G. Hong and L.P. Lee // Small6(4) (2010) 503.

[21] A.L. Kolesnikova, M.Yu. Gutkin and A.E. Romanov // Intern. J. Solids Structures 143 (2018) 59.

[22] H. Bateman and A. Erdélyi, Higher Transcendental Functions, vol. 1 (Mc GrawHill Book Company INC, New York, 1953).

[23] A.I. Lurie, Spatial problems of theory of elasticity (State Publishing House of Scientific and Technical Literature, Moscow, 1955), In Russian.

[24] T. Mura, Micromechanics of Defects in Solids (Martinus Nijhoff, Boston, 1975). 